\title{
$N$-Phenyl heteroarylamine analogues of fluazinam using the intermediate derivatization methods approach
}

\author{
AIYING GUAN ${ }^{\mathrm{a}}$, HUICHAO LI ${ }^{\mathrm{a}}$, ZHINIAN LI $^{\mathrm{a}}$, FAN YANG $^{\mathrm{a}}$, YONG XIE ${ }^{\mathrm{a}}$, XIAOPING YANG $^{\mathrm{b}}$ \\ and CHANGLING LIU ${ }^{\mathrm{a}, *}$

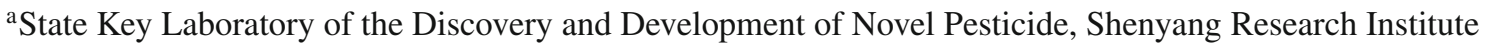 \\ of Chemical Industry Co. Ltd., Shenyang 110 021, China \\ ${ }^{\mathrm{b}}$ Division of Medical Oncology, Department of Medicine, School of Medicine, University of Colorado \\ Anschutz Medical Campus, Aurora, 80045, USA \\ e-mail: liuchangling@vip.163.com
}

MS received 12 November 2013; revised 11 April 2014; accepted 11 April 2014

\begin{abstract}
Twenty-one N-phenyl heteroarylamine analogues of fluazinam $3 \mathrm{a}-3 \mathrm{u}$ were prepared via nucleophilic substitution reaction of 2,6-dichloro-3,5-dinitrotoluene with heteroarylamines using the intermediate derivatization method. 2,6-Dichloro-3,5-dinitrotoluene, the key intermediate, was synthesized by nitration of 2,6-dichlorotoluene. The synthesized compounds were identified by ${ }^{1} \mathrm{H} \mathrm{NMR},{ }^{13} \mathrm{C} \mathrm{NMR}$, MS and elemental analyses. Preliminary bioassays indicated that most of the compounds showed good fungicidal activity against rice blast. The activity of $3 p$ was equal to that of fluazinam. The relationship between molecular structure and biological activity suggested that introduction of electron-withdrawing groups in the pyridine ring was important for optimizing fungicidal activity against rice blast.
\end{abstract}

Keywords. Diarylamine compounds; 2,6-Dichloro-3,5-dinitrotoluene; intermediate derivatization method; fungicides.

\section{Introduction}

A new approach for discovering and developing novel agrochemicals, Intermediate Derivatization Methods (IDM),${ }^{1-3}$ has been reported recently and was adopted by Shenyang Research Institute of Chemical Industry (SYRICI) to obtain innovative chemical structures which in conjunction with biological screening generated patentable leads and target compounds. ${ }^{4-9}$ The essence of IDM is to apply simple organic chemical reactions using commercially available and inexpensive starting materials to achieve innovated structures.

One application of IDM involves the use of 2,6dichlorotoluene as the key starting material for pesticides shown in figure 1, including herbicides dichlobenil (III), ${ }^{10}$ tembotrione (1-1) ${ }^{11}$ and florasulame(1-2), ${ }^{12}$ insecticides methoxyfenozide (1-3) ${ }^{13}$ and diflubenzuron (1-4),${ }^{10,14,15}$ and the fungicide fluopicolide (1-5) ${ }^{16}$ 2,6Dichlorotoluene was selected as the key starting material for these pesticides due to its suitable functionality

*For correspondence and its advantages of relatively low cost and wide commercial availability. We were interested in using the IDM approach with 2,6-dichlorotoluene as a starting material for the synthesis of analogues of the fluazinam, a phenylpyridinamine fungicide shown in figure 2 . Fluazinam shows good control on many diseases like grey mould and downy mildew on vines; apple scab, southern blight and white mould on peanuts; tomato late blight and tuber blight on potatoes; clubroot on crucifers; rhizomania on sugar beet; white and violet root rot on fruit trees; and for controlling mites in apples as well. ${ }^{17}$ In our previous study ${ }^{18}$ we used the IDM approach starting with 2,6dichlorotoluene to prepare the fluazinam analogue compound A (figure 2) in which $N$-(3-chloro-2,6-dinitro-4trifluoromethylphenyl) moiety of fluzinam was replaced by a $N$-(3-chloro-2-methyl-4,6-dinitrophenyl) group. This fluazinam analogue displayed promising control of rice blast and prompted us to explore similar analogues in order to improve its fungicidal activity. In this study, we report the synthesis of 21 new $N$ phenyl heteroarylamine analogues of fluazinam, prepared from key starting material 2,6-dichlorotoluene using the IDM approach, and their activity against rice blast. 


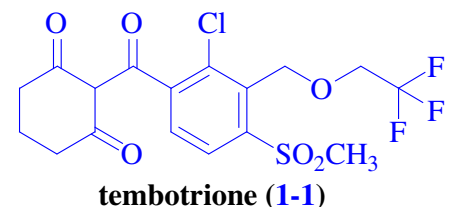<smiles>COc1ncc(F)c2nc(S(=O)(=O)Nc3c(F)cccc3F)nn12</smiles><smiles>O=C(NC(=O)c1c(F)cccc1F)Nc1ccc(Cl)cc1</smiles><smiles>CO[S+](=O)(OC)c1ccc(C(=O)O)c(Cl)c1COCC(F)(F)F</smiles><smiles>CC(=O)c1ccc(Cl)c(C)c1Cl</smiles>

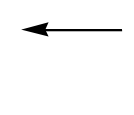<smiles>COc1cccc(Cl)c1C</smiles>

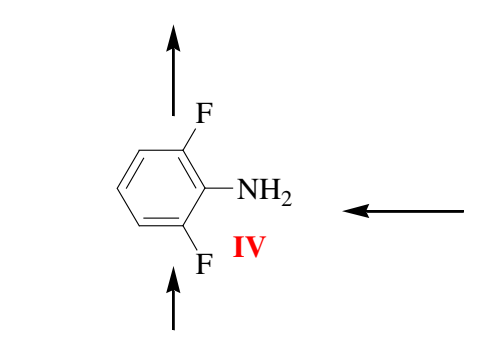

diflubenzuron (1-4)<smiles>COc1cccc(C#N)c1C</smiles>

II-A

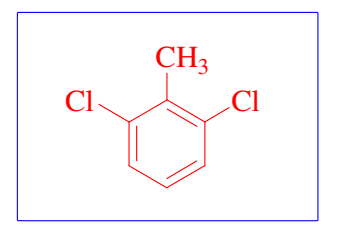

2,6-dichloro-toluene (1)

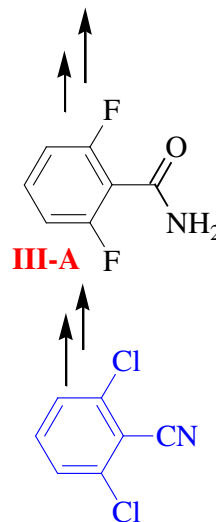

dichlobenil (III)<smiles>O=C(O)c1c(Cl)cccc1Cl</smiles>

fluopicolide (1-5)

Figure 1. Pesticides synthesized from key starting material 2,6-dichlorotoluene.

\section{Experimental section}

\subsection{Materials}

The reactions were monitored by TLC on aluminumbacked silica plate visualized by UV light. Melting points were determined on a Büchi melting point apparatus and are uncorrected. ${ }^{1} \mathrm{H}$ NMR spectra were recorded on a Mercury 300 (Varian, $300 \mathrm{MHz}$ ) spectrometer with deuterochloroform as the solvent and tetramethylsilane (TMS) as the internal standard. Mass<smiles>O=[N+]([O-])c1cc(C(F)(F)F)c(Cl)c([N+](=O)[O-])c1Nc1ncc(C(F)(F)F)cc1Cl</smiles>

Fluazinam<smiles>Cc1c(Cl)c([N+](=O)[O-])cc([N+](=O)[O-])c1Nc1ncc(C(F)(F)F)cc1Cl</smiles>

Compound A
Figure 2. Structures of fluazinam and compound A spectra were recorded using a JEOL JMS-700 mass spectrometer. Elemental analyses were determined on a Yanaco MT-3CHN elemental analyzer. All starting materials, reagents and solvents were commercially available and used without further purification.

\subsection{Synthesis of key intermediate 2,6-dichloro-3,5-} dinitrotoluene (2)<smiles>Cc1c(Cl)c([N+](=O)[O-])cc([N+](=O)[O-])c1Cl</smiles>

The synthesis sequence is outlined in scheme 1 . To a mixture of 2,6-dichlorotoluene $(50 \mathrm{mmol})$ and concentrated sulfuric acid $(30 \mathrm{~mL})$, cooled to $5^{\circ} \mathrm{C}$, was added fuming nitric acid ( $\mathrm{d}=1.52,10 \mathrm{~mL})$ dropwise for 20 min, while being stirred continuously. The reaction mixture was stirred at room temperature for an hour and then poured into icy water. The resulting pre- 


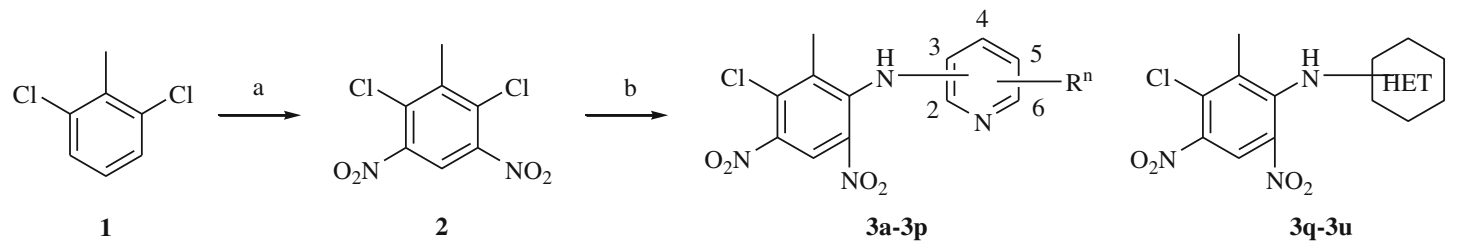

\begin{tabular}{|lll|}
\hline Pyridin-2-yl: & Pyridin-3-yl: & Pyridin-4-yl: \\
3a: $\mathrm{R}^{\mathrm{n}}=\mathrm{H} ;$ & 3g: $\mathrm{R}^{\mathrm{n}}=\mathrm{H} ;$ & 3n: $\mathrm{R}^{\mathrm{n}}=2-\mathrm{Cl} ;$ \\
3b: $\mathrm{R}^{\mathrm{n}}=4-\mathrm{CH}_{3} ;$ & 3h: $\mathrm{R}^{\mathrm{n}}=2-\mathrm{Cl} ;$ & 3o: $\mathrm{R}^{\mathrm{n}}=2-\mathrm{Br} ;$ \\
3c: $\mathrm{R}^{\mathrm{n}}=5-\mathrm{CH}_{3} ;$ & 3i: $\mathrm{R}^{\mathrm{n}}=2-\mathrm{Br} ;$ & 3p: $\mathrm{R}^{\mathrm{n}}=3-\mathrm{Cl}-2,5,6-\mathrm{F}_{3}$ \\
3d: $\mathrm{R}^{\mathrm{n}}=5-\mathrm{Cl} ;$ & 3j: $\mathrm{R}^{\mathrm{n}}=6-\mathrm{Cl} ;$ \\
3e: $\mathrm{R}^{\mathrm{n}}=5-\mathrm{Br} ;$ & 3k: $\mathrm{R}^{\mathrm{n}}=6-\mathrm{Br} ;$ \\
3f: $\mathrm{R}^{\mathrm{n}}=5-\mathrm{CF}_{3}$ & 3l: $\mathrm{R}^{\mathrm{n}}=2,5-\mathrm{Cl}_{2} ;$ \\
& 3m: $\mathrm{R}^{\mathrm{n}}=2,6-\mathrm{Cl}_{2}-4-\mathrm{CF}_{3}$ \\
\hline
\end{tabular}

Scheme 1. Reagents and conditions: (a) fuming nitric acid in c. $\mathrm{H}_{2} \mathrm{SO}_{4} / 5{ }^{\circ} \mathrm{C}$-room temperature; (b) heteroarylamines, $\mathrm{NaOH}$ in $\mathrm{DMF}$, room temperature $\left(25^{\circ} \mathrm{C}\right)$.

cipitate was collected by filtration, washed with water and recrystallized from ethanol giving 2,6-dichloro-3,5dinitrotoluene as pale green needles, M.p. $131-132^{\circ} \mathrm{C}$.

\subsection{General synthetic procedure for N-phenyl heteroamines $(\mathbf{3 a}-\mathbf{3 u})$}

The synthesis sequence is outlined in scheme 1 . Heteroarylamine $4(5 \mathrm{mmol})$ was added in portions to a suspension of $\mathrm{NaOH}(10 \mathrm{mmol}, 60 \%)$ in DMF $(20 \mathrm{~mL})$ at room temperature. After $30 \mathrm{~min}$, a solution of 2,6dichloro-3,5-dinitrotoluene $(6 \mathrm{mmol})$ in $30 \mathrm{~mL}$ of THF was added and then the resulting mixture was stirred overnight. After reaction completion (TLC), the mixture was filtered. The filtrate was concentrated under reduced pressure and the residue was purified on a silica column to afford the $N$-phenyl heteroarylamines $\mathbf{3 a - 3 u}$ in $60-91 \%$ yield. All these target compounds are new and are characterized as follows.

\subsection{Characterization of N-phenyl heteroarylamines $(3 a-3 u)$}

2.4a N-(3-chloro-2-methyl-4,6-dinitrophenyl)-5methylpyridin-2-amine (3c):<smiles>Cc1ccc(Nc2c([N+](=O)[O-])cc([N+](=O)[O-])c(Cl)c2C)nc1</smiles>

Yield 70\%. Orange solid. M.p.: $187-189^{\circ} \mathrm{C} ;{ }^{1} \mathrm{H}$ NMR $\left(300 \mathrm{MHz}, \mathrm{CDCl}_{3}\right)$ : $\delta$ (ppm) 9.04 (bs, 1H, NH), $8.62(\mathrm{~s}, 1 \mathrm{H}, \mathrm{Ph}-\mathrm{H}), 8.03$ (s, 1H, pyridin-6H), 7.50 (dd, $1 \mathrm{H}, J=2.4,8.4 \mathrm{~Hz}$, pyridin- $4 \mathrm{H}), 6.83(\mathrm{~d}, 1 \mathrm{H}$, $J=8.4 \mathrm{~Hz}$, pyridin-3H), 2.25 (s, 3H, Ph- $\left.\mathrm{CH}_{3}\right), 2.05$ (s, 3H, pyridin- $\left.\mathrm{CH}_{3}\right) ;{ }^{13} \mathrm{C}$ NMR $\left(75 \mathrm{MHz}\right.$, DMSO-d $\mathrm{d}_{6}$ ) $\delta$ 151.76, 146.32, 141.55, 140.42, 139.09, 137.04, 134.49, 129.94, 125.81, 120.64, 111.39, 17.22, 16.52; Anal. Calcd. for $\mathrm{C}_{13} \mathrm{H}_{11} \mathrm{ClN}_{4} \mathrm{O}_{4}: \mathrm{C}, 48.38 ; \mathrm{H}, 3.44 ; \mathrm{N}$, 17.36; Found: C, 48.31; H, 3.56; N, 17.31 .

2.4b 5-Chloro-N-(3-chloro-2-methyl-4,6-dinitrophenyl) pyridin-2-amine $(3 d)$ :<smiles>Cc1c(Cl)c([N+](=O)[O-])cc([N+](=O)[O-])c1Nc1ccc(Cl)cn1</smiles>

Yield 77\%. Orange solid. M.p.: $168-170^{\circ} \mathrm{C} ;{ }^{1} \mathrm{H}$ NMR $\left(300 \mathrm{MHz} \mathrm{CDCl}_{3}\right.$ ): $\delta$ (ppm) 8.89 (bs, 1H, NH), 8.59 (s, $1 \mathrm{H}, \mathrm{Ph}-\mathrm{H}), 8.13\left(\mathrm{~d}, 1 \mathrm{H}, J=2.7 \mathrm{H}_{Z}\right.$, pyridin- $\left.6 \mathrm{H}\right)$, $7.63\left(\mathrm{dd}, 1 \mathrm{H}, J=2.7,8.7 \mathrm{H}_{Z}\right.$, pyridin $\left.-4 \mathrm{H}\right), 6.86(\mathrm{~d}, 1 \mathrm{H}$, $J=8.7 \mathrm{H}_{\mathrm{Z}}$, pyridin-3H), $2.25\left(\mathrm{~s}, 3 \mathrm{H}, \mathrm{CH}_{3}\right)$; Anal. Calcd. for $\mathrm{C}_{12} \mathrm{H}_{8} \mathrm{Cl}_{2} \mathrm{~N}_{4} \mathrm{O}_{4}: \mathrm{C}, 42.00 ; \mathrm{H}, 2.35 ; \mathrm{N}, 16.33$; Found: C, 42.06; H, 2.46; N, 16.25.

2.4c 2-Chloro-N-(3-chloro-2-methyl-4,6-dinitrophenyl) pyridin-3-amine $(\mathbf{3 h})$ :<smiles>Cc1c(Cl)c([N+](=O)[O-])cc([N+](=O)[O-])c1Nc1cccnc1Cl</smiles>

Yield 75\%. Orange solid. M.p.: $136-138^{\circ} \mathrm{C} ;{ }^{1} \mathrm{H}$ NMR $\left(300 \mathrm{MHz}, \mathrm{CDCl}_{3}\right): \delta(\mathrm{ppm}) 8.88(\mathrm{bs}, 1 \mathrm{H}, \mathrm{NH}), 8.67(\mathrm{~s}$, $1 \mathrm{H}, \mathrm{Ph}-\mathrm{H}), 8.15$ (dd, $1 \mathrm{H}, J=1.5,4.8 \mathrm{H}_{\mathrm{Z}}$, pyridin- $\left.6 \mathrm{H}\right)$, $7.18-7.22(\mathrm{~m}, 1 \mathrm{H}$, pyridin-5H), $6.83(\mathrm{dd}, 1 \mathrm{H}, J=0.9$, $7.8 \mathrm{H}_{\mathrm{Z}}$, pyridin- $\left.4 \mathrm{H}\right), 2.20\left(\mathrm{~s}, 3 \mathrm{H}, \mathrm{CH}_{3}\right)$; Anal. Calcd. for $\mathrm{C}_{12} \mathrm{H}_{8} \mathrm{Cl}_{2} \mathrm{~N}_{4} \mathrm{O}_{4}$ : C, 42.00; H, 2.35; N, 16.33; Found: C, 42.04; H, 2.48; N, 16.26. 
2.4d 2-Bromo-N-(3-chloro-2-methyl-4,6-dinitrophenyl) pyridin-3-amine $(3 \boldsymbol{i})$ :<smiles>Cc1c(Cl)c([N+](=O)[O-])cc([N+](=O)[O-])c1Nc1cccnc1Br</smiles>

Yield 76\%. Orange solid. M.p.: $140-142{ }^{\circ} \mathrm{C} ;{ }^{1} \mathrm{H}$ NMR $\left(300 \mathrm{MHz}, \mathrm{CDCl}_{3}\right): \delta(\mathrm{ppm}) 8.89$ (bs, $\left.1 \mathrm{H}, \mathrm{NH}\right), 8.68$ (s, $1 \mathrm{H}, \mathrm{Ph}-\mathrm{H}), 8.13$ (dd, $1 \mathrm{H}, J=1.5,4.8 \mathrm{H}_{\mathrm{Z}}$, pyridin$6 \mathrm{H})$, 7.19-7.24 (m, 1H, pyridin-5H), $6.78(\mathrm{dd}, 1 \mathrm{H}$, $J=1.5,8.1 \mathrm{H}_{Z}$, pyridin-4H), $2.18\left(\mathrm{~s}, 3 \mathrm{H}, \mathrm{CH}_{3}\right)$; Anal. Calcd. for $\mathrm{C}_{12} \mathrm{H}_{8} \mathrm{BrClN}_{4} \mathrm{O}_{4}$ : C, 37.19; $\mathrm{H}, 2.08 ; \mathrm{N}, 14.46$; Found: C, 37.11; H, 2.14; N, 14.51.

2.4e 6-Chloro-N-(3-chloro-2-methyl-4,6-dinitrophenyl) pyridin-3-amine $(\mathbf{3 j})$ :<smiles>Cc1c(Cl)c([N+](=O)[O-])cc([N+](=O)[O-])c1Nc1ccc(Cl)nc1</smiles>

Yield $75 \%$. Orange solid. M.p.: $108-110{ }^{\circ} \mathrm{C} ;{ }^{1} \mathrm{H}$ NMR $\left(300 \mathrm{MHz}, \mathrm{CDCl}_{3}\right): \delta(\mathrm{ppm}) 9.07$ (bs, $\left.1 \mathrm{H}, \mathrm{NH}\right), 8.71$ (s, $1 \mathrm{H}, \mathrm{Ph}-\mathrm{H}), 8.05\left(\mathrm{~d}, 1 \mathrm{H}, J=2.7 \mathrm{H}_{Z}\right.$, pyridin- $\left.2 \mathrm{H}\right)$, $7.32\left(\mathrm{~d}, 1 \mathrm{H}, J=8.4 \mathrm{H}_{Z}\right.$, pyridin- $\left.5 \mathrm{H}\right), 7.15(\mathrm{dd}, 1 \mathrm{H}$, $J=2.7,8.4 \mathrm{H}_{Z}$, pyridin-4H), $2.18\left(\mathrm{~s}, 3 \mathrm{H}, \mathrm{CH}_{3}\right)$; Anal. Calcd. for $\mathrm{C}_{12} \mathrm{H}_{8} \mathrm{Cl}_{2} \mathrm{~N}_{4} \mathrm{O}_{4}$ : C, 42.00; $\mathrm{H}, 2.35 ; \mathrm{N}, 16.33$; Found: C, 41.93; H, 2.46; N, 16.24.

$2.4 \mathrm{f}$ 6-Bromo-N-(3-chloro-2-methyl-4,6-dinitrophenyl) pyridin-3-amine $(3 \boldsymbol{k})$ :<smiles>Cc1c(Cl)c([N+](=O)[O-])cc([N+](=O)[O-])c1Nc1ccc(Br)nc1</smiles>

Yield 75\%. Orange solid. M.p.: $108-110^{\circ} \mathrm{C} ;{ }^{1} \mathrm{H}$ NMR (300 MHz, $\mathrm{CDCl}_{3}$ ): $\delta$ (ppm) 9.03 (bs, $1 \mathrm{H}, \mathrm{NH}$ ), 8.71 (s, $1 \mathrm{H}, \mathrm{Ph}-\mathrm{H}), 8.04\left(\mathrm{~d}, 1 \mathrm{H}, J=3.0 \mathrm{H}_{Z}\right.$, pyridin- $\left.2 \mathrm{H}\right)$, $7.46\left(\mathrm{~d}, 1 \mathrm{H}, J=8.4 \mathrm{H}_{Z}\right.$, pyridin- $\left.5 \mathrm{H}\right), 7.04(\mathrm{dd}, 1 \mathrm{H}$, $J=3.0,8.4 \mathrm{H}_{Z}$, pyridin- $\left.4 \mathrm{H}\right), 2.18\left(\mathrm{~s}, 3 \mathrm{H}, \mathrm{CH}_{3}\right)$; Anal. Calcd. for $\mathrm{C}_{12} \mathrm{H}_{8} \mathrm{BrClN}_{4} \mathrm{O}_{4}$ : C, 37.19; H, 2.08; N, 14.46; Found: C, 37.14; H, 2.03; N, 14.57.

$2.4 \mathrm{~g}$ 2,5-Dichloro-N-(3-chloro-2-methyl-4,6-dinitrophenyl)pyridin-3-amine (3l):<smiles>Cc1c([N+](=O)[O-])cc(Cl)c(Cl)c1Nc1cc(Cl)cnc1Cl</smiles>

Yield 78\%. Orange solid. M.p.: $141-143^{\circ} \mathrm{C} ;{ }^{1} \mathrm{H}$ NMR $\left(300 \mathrm{MHz}, \mathrm{CDCl}_{3}\right): \delta(\mathrm{ppm}) 8.74$ (bs, $\left.1 \mathrm{H}, \mathrm{NH}\right), 8.65$ (s, 1H, Ph-H), 8.07 (d, $1 \mathrm{H}, J=2.1 \mathrm{H}_{Z}$, pyridin- $6 \mathrm{H}$ ), $6.72\left(\mathrm{~d}, 1 \mathrm{H}, J=2.1 \mathrm{H}_{Z}\right.$, pyridin- $\left.4 \mathrm{H}\right), 2.26(\mathrm{~s}$, $3 \mathrm{H}, \mathrm{CH}_{3}$ ). Anal. Calcd. for $\mathrm{C}_{12} \mathrm{H}_{7} \mathrm{Cl}_{3} \mathrm{~N}_{4} \mathrm{O}_{4}$ : C, 38.17; $\mathrm{H}, 1.87$; N, 14.84; Found: C, 38.25; H, 1.75; N 14.79 .

2.4h 2,6-Dichloro-N-(3-chloro-2-methyl-4,6-dinitrophenyl)-4-(trifluoromethyl)pyridin-3-amine $(\mathbf{3 m})$ :<smiles>Cc1c(Cl)c([N+](=O)[O-])cc([N+](=O)[O-])c1Nc1c(C(F)(F)F)cc(Cl)nc1Cl</smiles>

Yield 60\%. Yellow solid. M.p.: $130-132{ }^{\circ} \mathrm{C} ;{ }^{1} \mathrm{H}$ NMR $\left(300 \mathrm{MHz}, \mathrm{CDCl}_{3}\right): \delta(\mathrm{ppm}) 9.59(\mathrm{bs}, 1 \mathrm{H}, \mathrm{NH}), 8.74(\mathrm{~s}$, $1 \mathrm{H}, \mathrm{Ph}-\mathrm{H}), 7.67$ (s, $1 \mathrm{H}$, pyridin-H), $2.00\left(\mathrm{~s}, 3 \mathrm{H}, \mathrm{CH}_{3}\right)$. Anal. Calcd. for $\mathrm{C}_{13} \mathrm{H}_{6} \mathrm{Cl}_{3} \mathrm{~F}_{3} \mathrm{~N}_{4} \mathrm{O}_{4}$ : C, 35.04; $\mathrm{H}, 1.36$; N, 12.57; Found: C, 34.99; H, 1.44; N 12.52.

\section{4i 2-Bromo-N-(3-chloro-2-methyl-4,6-dinitrophenyl)} pyridin-4-amine (3o):<smiles>Cc1c(Cl)c([N+](=O)[O-])cc([N+](=O)[O-])c1Nc1ccnc(Br)c1</smiles>

Yield $79 \%$. Orange solid. M.p.: $170-172{ }^{\circ} \mathrm{C} ;{ }^{1} \mathrm{H}$ NMR $\left(300 \mathrm{MHz}, \mathrm{CDCl}_{3}\right): \delta(\mathrm{ppm}) 8.68(\mathrm{~s}, 1 \mathrm{H}$, pyridin-2H), 8.61 (bs, $1 \mathrm{H}, \mathrm{NH}), 8.59(\mathrm{~s}, 1 \mathrm{H}, \mathrm{Ph}-\mathrm{H}), 8.30(\mathrm{~d}, 1 \mathrm{H}$, $J=5.4 \mathrm{H}_{\mathrm{Z}}$, pyridin- $\left.6 \mathrm{H}\right), 6.26\left(\mathrm{~d}, 1 \mathrm{H}, J=5.4 \mathrm{H}_{\mathrm{Z}}\right.$, pyridin-5H), $2.30\left(\mathrm{~s}, 3 \mathrm{H}, \mathrm{CH}_{3}\right)$; Anal. Calcd. for $\mathrm{C}_{12} \mathrm{H}_{8} \mathrm{BrClN}_{4} \mathrm{O}_{4}$ : C, 37.19; H, 2.08; N, 14.46; Found: C, 37.14; H, 2.10; N, 14.50.

2.4j 3-Chloro-N-(3-chloro-2-methyl-4,6-dinitrophenyl)2,5,6-trifluoropyridin-4-amine (3p):<smiles>Cc1c(Cl)c([N+](=O)[O-])cc([N+](=O)[O-])c1Nc1c(F)c(F)nc(F)c1Cl</smiles>

Yield 91\%. Yellow Oil; ${ }^{1} \mathrm{H}$ NMR $\left(300 \mathrm{MHz}, \mathrm{CDCl}_{3}\right)$ : $\delta(\mathrm{ppm}) 8.54(\mathrm{~s}, 1 \mathrm{H}, \mathrm{Ph}-\mathrm{H}), 8.44$ (bs, 1H, NH), $2.33\left(\mathrm{~s}, 3 \mathrm{H}, \mathrm{CH}_{3}\right) ;{ }^{13} \mathrm{C} \mathrm{NMR}(75 \mathrm{MHz}, \mathrm{DMSO})$ $\delta 146.23,144.69,142.74,142.65,140.19,140.16$, $135.46,129.84,119.35,100.08(\mathrm{~d}, J=4.65 \mathrm{~Hz}), 99.51$, 16.35; Anal. Calcd. for $\mathrm{C}_{12} \mathrm{H}_{5} \mathrm{Cl}_{2} \mathrm{~F}_{3} \mathrm{~N}_{4} \mathrm{O}_{4}$ : C, 36.30; $\mathrm{H}$, 1.27; N, 14.11; Found: C, 36.38; H, 1.22; N, 14.15; LC-MS ( $m / z \%): 394.9$ [M-H] $]^{-}$. 
2.4k N-(3-chloro-2-methyl-4,6-dinitrophenyl)pyrimidin2-amine $(3 \mathrm{~s})$ :<smiles>Cc1c(Cl)c([N+](=O)[O-])cc([N+](=O)[O-])c1Nc1ncccn1</smiles>

Yield 83\%. Yellow solid. M.p.: $108-110^{\circ} \mathrm{C} ;{ }^{1} \mathrm{H}$ NMR $\left(300 \mathrm{MHz}, \mathrm{CDCl}_{3}\right): \delta(\mathrm{ppm}) 8.67$ (bs, $\left.1 \mathrm{H}, \mathrm{NH}\right), 8.49$ (s, $1 \mathrm{H}, \mathrm{Ph}-\mathrm{H}), 8.44\left(\mathrm{~d}, 2 \mathrm{H}, J=4.8 \mathrm{H}_{\mathrm{Z}}\right.$, pyrimidin- $\left.4,6-2 \mathrm{H}\right)$, $6.89-6.93\left(\mathrm{~m}, 1 \mathrm{H}\right.$, pyrimidin-5H), $2.43\left(\mathrm{~s}, 3 \mathrm{H}, \mathrm{CH}_{3}\right)$; ${ }^{13} \mathrm{C}$ NMR (75 MHz, DMSO) $\delta 159.50,158.29,144.00$, 142.93, 137.54, 135.59, 129.87, 119.94, 114.02, 16.57; Anal. Calcd. for $\mathrm{C}_{11} \mathrm{H}_{8} \mathrm{ClN}_{5} \mathrm{O}_{4}: \mathrm{C}, 42.66 ; \mathrm{H}, 2.60 ; \mathrm{N}$, 22.62; Found: C, 42.62; H, 2.56; N, 22.67.

\section{$2.41 \quad N$-(3-chloro-2-methyl-4,6-dinitrophenyl)thiazol-} 2-amine (3t):<smiles>CC(C)(C)c1c(Cl)c([N+](=O)[O-])cc([N+](=O)[O-])c1Nc1nccs1</smiles>

Yield $71 \%$. Orange solid. M.p.: $151-153{ }^{\circ} \mathrm{C} ;{ }^{1} \mathrm{H}$ NMR $\left(300 \mathrm{MHz}, \mathrm{CDCl}_{3}\right): \delta$ (ppm) 9.34 (bs, $\left.1 \mathrm{H}, \mathrm{NH}\right), 8.60$ (s, 1H, Ph-H), $7.28(\mathrm{~d}, 1 \mathrm{H}, J=3.6 \mathrm{~Hz}$, thiazol-4-H), $6.93\left(\mathrm{~d}, 1 \mathrm{H}, J=3.6 \mathrm{~Hz}\right.$, thiazol-5H), $2.31\left(\mathrm{~s}, 3 \mathrm{H}, \mathrm{CH}_{3}\right)$; Anal. Calcd. for $\mathrm{C}_{10} \mathrm{H}_{7} \mathrm{ClN}_{4} \mathrm{O}_{4} \mathrm{~S}$ : C, 38.16; H, 2.24; N, 17.80; Found: C, 38.19; H, 2.30; N, 17.68.

\subsection{Fungicidal assays}

The in vitro biological activities were evaluated using a previously reported procedure ${ }^{18}$ with the aid of High Throughput Screening. A testing solution was obtained by dissolving each $N$-phenyl heteroarylamine compound in various solvents at the desired concentration, ranging from $0.1-25 \mathrm{mg} / \mathrm{L}$. The solvents were selected from acetone, methanol or DMF according to the different solubility of each compound. The testing solution and pathogen suspensions were added into the cells of a 96-well culture plate, which then was placed in the constant temperature chamber. After $24 \mathrm{~h}$ pathogen germination or growth was assessed and the fungicidal activity of the compound was evaluated relative to pathogen germination or growth in the control treatment. The level of fungicidal activity was reported in the range 0 (no control) to $100 \%$ (complete control).

\section{Results and discussion}

\subsection{Chemistry}

The preparation of target compounds $\mathbf{3 a}-\mathbf{3} \mathbf{u}$ illustrated in scheme 1, was accomplished by adopting known methods. ${ }^{18-20}$ The intermediate 2,6-dichloro-3,5-dinitrotoluene (2) was prepared from 2,6-dichlorotoluene (1) and fuming nitric acid, then reacted with heteroaryl amines (4) to afford target compounds (3) in 60-91\% yields. The $21 \mathrm{~N}$-phenyl heteroarylamine compounds are listed in table 1.

\subsection{Structure-activity relationship (SAR) based on results of in vitro fungicide assay}

The compounds were screened for fungicidal activity against rice blast $(\mathrm{RB})$, a major pathogen of rice. As shown in table 2, all $N$-phenyl heteroarylamine compounds, except 3c, displayed significant fungicidal activity against RB. Compounds $\mathbf{3 m}$ and $\mathbf{3 p}$ were particularly effective, providing $80 \%$ and $100 \%$ control respectively, at the lowest concentration, $0.1 \mathrm{mg} / \mathrm{L}$ and were much improved over that of compound $\mathbf{A}$ ( $80 \%$ at $25 \mathrm{mg} / \mathrm{L}$ ) and equal to that of fluazinam. Based on the data presented in table 2 , a structure-activity relationship emerged.

3.2a Compounds of the 2-pyridinyl type $(3 a-3 f)$ : When electron-donating group such as methyl group was introduced at the 5-position of the pyridine ring in the 2-pyridinyl to form $\mathbf{3 c}$, fungicidal activity reduced compared to the unsubstituted pyridine compound $\mathbf{3 a}$ (50\% vs. $100 \%$ control at $25 \mathrm{mg} / \mathrm{L})$. When the methyl group was introduced at the 4-position of the pyridine ring giving $\mathbf{3 b}$, fungicidal activity remained the same as the unsubstituted pyridine compound 3a ( $80 \%$ control at $8.3 \mathrm{mg} / \mathrm{L}$ ). This result suggests that the introduction of methyl at 4-position would not improve fungicidal activity in this series of $N$-phenylpyridinamines. However, when the 5-position was substituted with an electron-withdrawing group (chlorine, bromine or trifluoromethyl), the fungicidal activity of compounds 3d, 3e and 3f was improved over the unsubstituted pyridine compound 3a (100\%, 100\%, 100\% vs. $80 \%$ at $8.3 \mathrm{mg} / \mathrm{L}$ ). These results imply that an electronwithdrawing group at the 5-position of the pyridine ring improves fungicidal activity.

3.2b Compounds of the 3-pyridinyl type $(3 g-3 m)$ : Introducing a chlorine atom at the 2- or 6-position of pyridine ring in the 3-pyridinyl type of compounds to form $\mathbf{3 h}$ and $\mathbf{3} \mathbf{j}$, fungicidal activity was improved compared with the unsubstituted pyridine compound $3 g(80 \%, 80 \%$ vs $0 \%$ at $2.8 \mathrm{mg} / \mathrm{L})$. Similarly, introducing bromine at the 2- or 6-position to form $3 \mathbf{i}$ and $3 \mathbf{k}$ also improved fungicidal activity compared to the unsubstituted pyridine compound $\mathbf{3 g}$ (100\%, $80 \%$ vs $0 \%$ at $2.8 \mathrm{mg} / \mathrm{L})$. When hydrogen atoms at the 2 - and 
Table 1. Chemical structures, physical properties of compounds synthesized.

\begin{tabular}{|c|c|c|c|c|}
\hline Compd. & $\begin{array}{c}\text { Position of } \\
-\mathrm{NH}^{\mathrm{a}}{ }^{\mathrm{a}}\end{array}$ & $\mathrm{R}^{n}$ & M.p. $\left({ }^{\circ} \mathrm{C}\right)$ & Yield (\%) \\
\hline $3 \mathbf{a}$ & 2 & $\mathrm{H}$ & $111-113$ & 73 \\
\hline $\mathbf{3 b}$ & 2 & $4-\mathrm{CH}_{3}$ & $150-152$ & 68 \\
\hline $3 \mathbf{c}$ & 2 & $5-\mathrm{CH}_{3}$ & $187-189$ & 70 \\
\hline 3d & 2 & $5-\mathrm{Cl}$ & $168-170$ & 77 \\
\hline $3 \mathbf{e}$ & 2 & $5-\mathrm{Br}$ & $175-177$ & 79 \\
\hline 3f & 2 & $5-\mathrm{CF}_{3}$ & $142-144$ & 65 \\
\hline $3 \mathbf{g}$ & 3 & $\mathrm{H}$ & $146-148$ & 78 \\
\hline $3 \mathbf{h}$ & 3 & $2-\mathrm{Cl}$ & $136-138$ & 75 \\
\hline 3i & 3 & $2-\mathrm{Br}$ & $140-142$ & 76 \\
\hline $3 \mathbf{j}$ & 3 & $6-\mathrm{Cl}$ & $108-110$ & 75 \\
\hline 3k & 3 & $6-\mathrm{Br}$ & $156-158$ & 78 \\
\hline $3 \mathbf{I}$ & 3 & $2,5-\mathrm{Cl}_{2}$ & $141-143$ & 78 \\
\hline $3 \mathbf{m}$ & 3 & $2,6-\mathrm{Cl}_{2}-4-\mathrm{CF}_{3}$ & $130-132$ & 60 \\
\hline $3 \mathbf{n}$ & 4 & $2-\mathrm{Cl}$ & $166-168$ & 80 \\
\hline 30 & 4 & $2-\mathrm{Br}$ & $170-172$ & 79 \\
\hline $3 p$ & 4 & $3-\mathrm{Cl}-2,5,6-\mathrm{F}_{3}$ & oil & 91 \\
\hline Compd. & & & M.p. $\left({ }^{\circ} \mathrm{C}\right)$ & Yield (\%) \\
\hline $3 q$ & & & $175-177$ & 80 \\
\hline $3 \mathbf{r}$ & & & $108-110$ & 74 \\
\hline $3 s$ & & & $178-180$ & 83 \\
\hline $3 t$ & & & $151-153$ & 71 \\
\hline $3 \mathbf{u}$ & & & $137-139$ & 69 \\
\hline
\end{tabular}

ameans that "-NH-" is attached to pyridinyl ring.

5-position of pyridine ring were simultaneously substituted with chlorine atoms (3I), its activity was further enhanced compared with that of compounds $\mathbf{3 h}, \mathbf{3 i}, \mathbf{3 j}$ and 3k $(50 \%$ vs. $0 \%$ control at $0.9 \mathrm{mg} / \mathrm{L})$. Astonishingly, the trisubstituted pyridine compound $\mathbf{3 m}$ which possesses electron-withdrawing groups $2,6-\mathrm{Cl}_{2}-4-\mathrm{CF}_{3}$ provided greatly improved fungicidal activity compared to the unsubstituted pyridine compound $\mathbf{3 g}$ ( $80 \%$ vs. no control at $0.1 \mathrm{mg} / \mathrm{L}$ ), this result suggests that the strong electron-withdrawing $\mathrm{CF}_{3}$ on 4-position of pyridine ring plays an important role in enhancing fungicidal activity in combination with $2,6-\mathrm{Cl}_{2}$. It is not difficult to draw the conclusion that the adjacent, and particularly para positions of nitrogen atom in pyridine ring has a significant effect on fungicidal activity.

3.2c Compounds of the 4-pyridinyl type $(3 \boldsymbol{n}-3 p)$ : Introducing a chlorine or bromine atom at the 2-position of the pyridine ring forming $\mathbf{3 n}$ and $\mathbf{3 o}$ ) gave $80 \%$ control at $8.3 \mathrm{mg} / \mathrm{L}$ and $80 \%$ control at $2.8 \mathrm{mg} / \mathrm{L}$, respectively. In this case, the bromine compound showed superior activity compared with the corresponding compound containing chlorine. The tetrasubstituted pyridine compound $\mathbf{3 p}$ which includes electronwithdrawing atoms $3-\mathrm{Cl}-2,5,6-\mathrm{F}_{3}$ displayed the highest fungicidal activity of all tested $N$-phenyl heteroarylamine compounds ( $100 \%$ control at $0.1 \mathrm{mg} / \mathrm{L})$, which may be due to strong electronegativity of fluorine and/or multiple substituents.

3.2d Other nitrogen heterocyclic analogues (3q $3 \boldsymbol{u})$ : Replacing the 2-pyridinyl ring of 3a with the 3isoxazolyl ring forming $3 \mathrm{u}$ reduced fungicidal activity (50\% control at $8.3 \mathrm{mg} / \mathrm{L}$ ). When the 2-pyridinyl ring of 3a was replaced with a 2-pyrazinyl or 2-pyrimidinyl ring forming compounds $3 \mathrm{r}$ and $3 \mathrm{~s}$, fungicidal activity was maintained $(80 \%$ control at $8.3 \mathrm{mg} / \mathrm{L})$. When the 2-pyridinyl ring of $3 \mathrm{a}$ was replaced with a 2-quinolinyl 
Table 2. Fungicidal activity of $N$-phenyl heteroarylamines against RB.

\begin{tabular}{|c|c|c|c|c|c|c|}
\hline \multirow[b]{2}{*}{ Compd. } & \multicolumn{6}{|c|}{$\begin{array}{c}\text { Fungicidal activity against RB } \\
\text { (\% control at given concentration } \mathrm{mg} / \mathrm{L} \text { ) }\end{array}$} \\
\hline & 25 & 8.3 & 2.8 & 0.9 & 0.3 & 0.1 \\
\hline $3 \mathbf{a}$ & 100 & 80 & 0 & /b & I & I \\
\hline $3 \mathbf{b}$ & 100 & 80 & 0 & I & l & I \\
\hline $3 c$ & 50 & 1 & I & I & I & I \\
\hline 3d & 100 & 100 & 50 & 0 & l & I \\
\hline $3 \mathbf{e}$ & 100 & 100 & 50 & 0 & I & l \\
\hline 3f & 100 & 100 & 50 & 0 & I & I \\
\hline $3 g$ & 100 & 50 & 0 & I & 1 & l \\
\hline $3 \mathbf{h}$ & 100 & 100 & 80 & 0 & I & l \\
\hline $3 \mathbf{i}$ & 100 & 100 & 100 & 0 & I & I \\
\hline $3 \mathbf{j}$ & 100 & 100 & 80 & 0 & I & I \\
\hline 3k & 100 & 100 & 80 & 0 & 1 & I \\
\hline 31 & 100 & 80 & 80 & 50 & 1 & I \\
\hline $3 \mathbf{m}$ & 100 & 100 & 100 & 100 & 100 & 80 \\
\hline 3n & 100 & 80 & 0 & I & I & I \\
\hline 30 & 100 & 100 & 80 & 0 & I & I \\
\hline $3 \mathbf{p}$ & 100 & 100 & 100 & 100 & 100 & 100 \\
\hline $\mathbf{3 q}$ & 100 & 100 & 80 & 0 & I & I \\
\hline $3 \mathbf{r}$ & 100 & 80 & 0 & I & l & I \\
\hline $3 \mathrm{~s}$ & 100 & 80 & 0 & I & I & I \\
\hline $3 t$ & 100 & 100 & 100 & 0 & I & l \\
\hline 3u & 100 & 50 & 0 & I & l & I \\
\hline Compound A & 80 & 0 & I & I & 1 & I \\
\hline Fluazinam & 100 & 100 & 100 & 100 & 100 & 100 \\
\hline
\end{tabular}

$\mathrm{b}_{\text {stands for no data. }}$

ring forming $3 \mathrm{q}$, fungicidal activity increased $(80 \%$ control at $2.8 \mathrm{mg} / \mathrm{L}$ ). When the 2-pyridinyl ring of $3 \mathrm{a}$ was replaced with a 2 -thiazolyl ring forming $3 \mathrm{t}$, fungicidal activity increased further $(100 \%$ control at $2.8 \mathrm{mg} / \mathrm{L}$ ). These results suggest that further structure optimization is possible outside the pyridinyl type analogues, as shown by good activity found with the quinolinyl and thiazolyl analogues.

\section{Conclusions}

Twenty one $N$-phenyl heteroarylamine compounds were synthesized via the intermediate derivatization method. Overall, introduction of electronwithdrawing groups to any position of pyridine ring was important to optimize fungicidal activity against rice blast. Activity was generally greater in 3- and 4-pyridinyl type analogues compared with the 2pyridinyl type compounds and was improved when the number of electron-withdrawing substituents increased. Analogues of the non-pyridinyl type also showed promising fungicidal activity, particularly the quinolinyl and thiazolyl analogues. Further synthesis and structure optimization studies are in progress.

\section{Supplementary Information}

The electronic supporting information, the spectral data for compounds $\mathbf{3 a}, \mathbf{3 b}, \mathbf{3 e}, \mathbf{3 f}, \mathbf{3 g}, \mathbf{3 n}, \mathbf{3 q}, \mathbf{3 r}$ and $\mathbf{3 u}$, can be seen in www.ias.ac.in/chemsci.

\section{Acknowledgements}

We thank Dr. Mark Dekeyser (Canada) for assisting in preparing the manuscript. The project was supported by the National Key Technology Support Program during the 12th Five-Year Plan Period of China (Grant No. 2011BAE06B05).

\section{References}

1. Liu C L 2009 Frontiers of modern chemical engineering, metallurgy, and material technologies-7th Academic Conference of Chemical, J K Wang (ed.), (Beijing: Chemical Industry Press Publishers) p 86

2. Liu C L 2008 High Technol. Industrial 979

3. Liu C L 2011 Chin. J. Pestic. 5020

4. Guan A Y, Liu C L, Li M, Zhang H, Li Z N and Li Z M 2011 Pest Manag. Sci. 67647

5. Chai B S, Liu C L, Li H C, He X M, Luo Y M, Huang G, Zhang H and Chang J B 2010 Pest Manag. Sci. 66 1208 
6. Chai B S, Liu C L, Li H C, Zhang H, Liu S W, Huang G and Chang J B 2011 Pest Manag. Sci. 671141

7. Li M, Liu C L, Li L, Yang H, Li Z N, Zhang H and Li Z M 2010 Pest Manag. Sci. 66107

8. Li M, Liu C L, Zhang J, Wu Q, Hao S L and Song Y Q 2013 Pest Manag. Sci. 69635

9. Guan A Y, Liu C L, Huang G, Li H C, Hao S L, $\mathrm{Xu} \mathrm{Y}$ and Li Z N 2013 J. Agric. Food Chem. 61 11929

10. Martin A, Kalevaru V N and Smejkal Q 2010 Catal. Today $\mathbf{1 5 7} 275$

11. Almsick A, Benet-Buchhola J, Olenik B and Willms L 2009 Bayer CropScience Journal 625

12. Thompson A R, McReath A M, Carson C M, Ehr R J and DeBoer G J 1999 Brighton Conference-Weeds, (Brighton: UK) 1 p 73
13. Le D P, Thirugnanam M, Lidert Z, Carlson G R and Ryan J B 1996 Brighton Crop Protection ConferencePests and Diseases, (Brighton, UK) 2 p 481

14. Wang Y, Wei H W, Chen G Y, Pan M S and Miao Y C.N. patent 101503372, 2009

15. Wang Y L and Shi W B C N patent 102180813, 2011

16. Tafforeau S, Wegmann T, Latorse M P, Gouot J M, Duvert P and Bardsley E 2005 BCPC International Congress, Crop Science \& Technology, (Glasgow, UK) $1 \mathrm{p} 79$

17. Tomlin C D S 2009 The Pesticide Manual 15th ed., (Alton: BCPC) p 513

18. Guan A Y, Li H C, Li Z N, Hao S L, Lan J and Liu C L C.N. Patent 102827072, 2012

19. Suzuki H Tokkyo Koho Jpn. JP 2003292476, 2003

20. Ritter J C U.S. Patent 8183418, 2012 